\title{
Experimental study of nanofluids flow and heat transfer over a backward-facing step channel
}

\begin{abstract}
The forced convective heat transfer and friction factor of nanofluids flow over a backward-facing step (BFS) in a channel with the base wall dissipating a uniform heat flux are experimentally investigated in this paper. Nanoparticles such as $\mathrm{CuO}$ and $\mathrm{MgO}$ dispersed in pure ethylene glycol (EG) with average diameters of $40 \mathrm{~nm}$, and volume concentrations of $0,1,3$, and $5 \%$ are considered as the working nanofluids. The results indicate that the heat transfer rate increases as the volume concentration of nanoparticles increased. The Nusselt number is enhanced up to $11 \%$ at 0.05 volume concentration compared to pure EG. The friction factor increases up to approximately $15 \%$ at Reynolds number (Re) of 5000 and volume concentrations of 0.01 and 0.03 . The maximum value of the PEC in a BFS channel is 1.5 for the CuO-EG and 1.2 for the MgO-EG at a volume concentrations of 0.03 and $\operatorname{Re}=20,000$.
\end{abstract}

Keyword: $\mathrm{CuO}$ nanoparticles; Forced convection; Backward-facing step; Experimental Nusselt number 